\title{
Revisiting For Resolving: The Ecological Perspective of Periodontal Bacteriome for Prevention and Control of Periodontitis in COVID-19 Era
}

\author{
Perera $\mathrm{L}^{1 *}$ and Perera $\mathrm{I}^{2}$ \\ ${ }^{1}$ School of Dentistry \& Oral Health, Griffith University, Australia \\ ${ }^{2}$ Preventive Oral Health Unit, The National Dental Hospital, Sri Lanka
}

*Corresponding author: Lakmali Perera, School of Dentistry \& Oral Health, Gold Coast Campus, Griffith University, Australia, Tel: +94 71 3271945; Email: thumbapanni470723@ gmail.com

\section{Short Communication}

Volume 5 Issue 4

Received Date: October 12, 2020

Published Date: October 23, 2020

DOI: $10.23880 /$ ajds- 16000275

\section{Abstract}

Chronic periodontitis is a major public health challenge accounting for global burden of oral diseases. It has resulted in 3.5 million years lived with disability, 54-billion USD/year in lost productivity and a major portion of the 442 billion USD/year cost incurred for managing oral diseases. Nevertheless there is promising emerging evidence on prevention and control of periodontal disease as well as for the improvement of periodontal health by non-surgical periodontal therapy comprised of behavioural management for effective tooth brushing, flossing and maintaining optimal oral hygiene, habit intervention and the administration of probiotics, scaling treatment especially for patients suffering from mild to moderate periodontitis. In microbial ecological point of view, chronic period ontitis is a polymicrobial chronic inflammatory disease subjected to succession which is considered to be a major ecological event. Furthermore, risk/predisposing factors of periodontitis are responsible for creating ecological stress on normal sub gingival microbial flora. Thus, the objective of this short communication is to explain the rationale for potential success of non-surgical treatment modalities of management of periodontitis and establishing periodontal health by incorporating the ecological perspective of periodontitis. Further, this short communication highlights the prime importance of precision periodontal management and optimized treatment outcomes from molecular perspectives through to public health perspectives in the era of personalized/precision medicine impacted by COVID-19 pandemic as concluding remarks.

Keywords: Chronic periodontitis; Periodontal Bacteriome; Gingivitis and Periodontitis; Oral microbiome

\section{Introduction}

Gingivitis and Periodontitis are the most common inflammatory diseases of the periodontium/tooth supporting structures [1,2]. Clinically, periodontitis could be categorized into mild, moderate and severe forms depending on the severity of the disease. Unfortunately, chronic periodontitis contributes significantly to the global burden of oral diseases amounting for 3.5 million years lived with disability, 54 billion USD/year in lost productivity and a major portion of the 442 billion USD/year cost incurred for managing oral diseases [1]. Fortunately, there is accumulating evidence on the improvement of periodontal health by non-surgical periodontal therapy comprised of behavioural management for effective tooth brushing, flossing and maintaining optimal oral hygiene, habit intervention [2,3] and the administration of probiotics, scaling treatment, rational temporary use of anti-bacterial mouth rinses especially for patients suffering 


\section{Open Access Journal of Dental Sciences}

from mild to moderate periodontitis 3 . This indicates that the control of periodontitis could be accomplished by restoration of the healthy supra gingival and sub gingival oral microbiome, underpinned by maintenance of optimal oral hygiene which can be considered as a microbial intervention. The rationale for this important assumption could be succinctly explained by revisiting the ecological perspective of periodontal bacteriome [4].

Periodontitis is of poly microbial aetiology [5]. Poor oral hygiene, smoking, betel chewing with or without tobacco, genetic susceptibility, poor nutrition, age and immunosuppression of patients due to diabetes, pregnancy related hormonal changes. HIV infection are among the leading risk/predisposing factors of periodontitis [2]. Same of those factors can be considered as environmental factors which might induce an 'ecological stress' on normal sub gingival microbiome, when considering the ecological perspective. Therefore, in periodontitis a 'disturbance' or dysbiotic [6] state that disturbs the harmony of normal sub gingival micro flora occurs, facilitating the 'community assembly 'or enrichment of Gram negative, anaerobic periodonto pathogens in the sub gingival biofilm [7]. Hence, periodontal diseases are initiated and progressed by dysbiotic but metabolically compatible microbial consortia at or below the gingival margin. Virulent attributes of these microbes are termed as 'poly microbial synergy' involving concerted action, which could progress the disease condition via chronic inflammation [7]. This may get deteriorated with deep periodontal pocket formation, loss of attachment as well as loss of the alveolar bone around the teeth resulting in subsequent loss of teeth, if untreated [2]. Furthermore, 'mal odor' produced by 'inflammophilic' community enriched by Gram negative anaerobes which nourishes through inflammatory tissue breakdown-derived nutrients could be considered as the most devastating 'social issue' in untreated chronic periodontitis [8].

Succession is an ecological event which facilitates the periodontal community assembly [9]. This can be seen in the progression of periodontitis. Initial periodontal colonizers start flourishing in the supra gingival biofilm adhering to each other. They use strong short- range stereo chemical interactions between adhesions on the bacterium and complementary receptors to form distinct polymicrobial consortia. Hence, periodontitis associated meta community will integrate physically and physiologically to use wider range of nutritional substrates than a single species. Succession will follow when primary colonizers of a habitat facilitate the success of secondary colonizers. Succession of bacterial species in supragingival plaque samples was observed within hours, days and months $[9,10]$ of undisturbed plaque accumulation using biofilm technologies previously. The yellow complex facultative anaerobes such as Streptococcus intermedius, Streptococcus oralis and Streptococcus mitis were increased in numbers and proportions [8-10]. Then the consortium was expanded with purple complex member-Veillonella parvula, green complex member- Capnocytophaga gingivalis as well as an orange complex member-Fusobacterium nucleatum during 7-days of supra gingival biofilm regrowth, in the same experiment [8-10]. Finally, the red complex patho bionts Porphyromonas gingivalis, Tannerella forsythia and Treponema denticola were established in the supra gingival plaque to accomplish the meta community assembly. Transition from facultative anaerobes to strict anaerobes was evident in the progression of periodontitis, thus it is reasonable to assume that the hypoxic environment in the supra gingival biofilm is ideal for the colonization of strict anaerobes at the final stage of supra gingival biofilm [10]. Obviously, restoration of normal supra gingival and sub-gingival biofilms could be accomplished by non-surgical periodontal therapy.

Against this backdrop, microbial ecological studies on polymicrobial diseases emerge as a promising field of research, especially in the era of personalized or precision medicine. These studies will provide information on shifts of oral microbiome in oral and non-oral inflammatory diseases as applied to individuals. Findings of such research will shed light into precision periodontal disease management and optimized treatment outcomes from molecular perspectives through to public health perspectives. Optimal plaque control accomplished by effective tooth brushing and flossing with rational temporary use of anti-bacterial mouth rinses will cure gingivitis and facilitate maintenance of optimal oral hygiene that promotes establishment of healthy supra gingival and sub gingival microbiome. This becomes pertinent in the era of COVID-19 pandemic demonstrating devastating waves of spreading infection with increased virulence of the virus across the globe that had indelibly impacted on health and oral health care provision. Dental treatment are among high risk treatment procedures for spread of COVID-19 infection and ultrasonic scaling treatment to remove calculus deposits which generates aerosols is generally contraindicated during the rapid community spread of COVID-19 [11]. Hence, the rational use of fundamental of ecological perspectives of periodontium would be a potential pragmatic and innovative approach to prevention and control of periodontitis in the era of devastating COVID-19 pandemic.

\section{References}

1. Tonetti MS, Jepsen S, Jin L, Otomo Corgel J (2017) Impact of the global burden of periodontal diseases on health, nutrition and wellbeing of mankind: A call for global action. Journal of clinical periodontology 44(5): 456- 
462.

2. Khan SA, Kong EF, Meiller TF, Jabra Rizk MA (2015) Periodontal diseases: bug induced, host promoted. PLoS pathogens. 11(7): e1004952.

3. Donos N, Suvan JE, Calciolari E, Nibali, L, Rollnick S (2020) The effect of a behavioural management tool in adults with mild to moderate periodontitis. A singleblind, randomized controlled trial. J Periodont Res.

4. Haruta S, Saito Y, Futamata H (2016) Development of Microbial Ecological Theory: Stability, Plasticity, and Evolution of Microbial Ecosystems. Frontiers in microbiology 7: 2069.

5. Perera M, Al hebshi NN, Speicher DJ, Perera I, Johnson NW (2016) Emerging role of bacteria in oral carcinogenesis: a review with special reference to perio-pathogenic bacteria. J Oral Microbiol 8(1): 32762.

6. Lamont RJ, Hajishengallis G (2015) Polymicrobial synergy and dysbiosis in inflammatory disease. Trends in Molecular Medicine 21(3): 172-183.
7. Kilian M, Chapple I, Hannig M, Marsh P, Meuric V, et al. (2016) The oralmicrobiome-an update for oral healthcare professionals. British Dental Journal 221(10): 657-666.

8. Hajishengallis G, Lamont RJ (2012) Beyond the red complex and into more complexity: the polymicrobial synergy and dysbiosis (PSD) model of periodontal disease etiology. Mol Oral Microbiol 27(6): 409-419.

9. Teles F, Teles R, Sachdeo A, Uzel N, Song X, et al. (2012) Comparison of microbial changes in early redeveloping biofilms on natural teeth and dentures. J Periodontol 83(9): 1139-1148.

10. Uzel NG, Teles FR, Teles RP, Song XQ, Torresyap G, et al. (2011) Microbial shifts during dental biofilm re-development in the absence of oral hygiene in periodontal health and disease. J Clin Periodontol 38(7): 612-620.

11. Meng L, Hua F, Bian Z (2020) Coronavirus Disease 2019 (COVID-19): Emerging and Future Challenges for Dental and Oral Medicine. J Dent Res 99(5): 481-487. 